\title{
A Model to Realise Sustainability in Networked Production and Transportation
}

\author{
Giuseppe Stecca ${ }^{1}$, Giacomo Liotta ${ }^{2}$, and Toshiya Kaihara ${ }^{3}$ \\ ${ }^{1}$ Institute of Industrial Technologies and Automation, \\ National Research Council of Italy, Strada della Neve, Via Salaria Km. 29,300, \\ 00010 Montelibretti (Rome), Italy \\ giuseppe.stecca@itia.cnr.it \\ ${ }^{2}$ Department of Technology and Innovation, University of Southern Denmark, \\ Campusvej 55, DK-5230 Odense, Denmark \\ gileiti.sdu.dk \\ ${ }^{3}$ Graduate School of System Informatics, Kobe University, 1-1, \\ Rokkodai, Nada, 657- 8501 Kobe, Japan \\ kaihara@kobe-u.ac.jp
}

\begin{abstract}
Cost optimisation in decentralised, make-to-order production systems requires tight integration with suppliers and transport operations as well as flexible network connections. Additionally, minimisation of environmental impacts of freight flows must be included in the industrial practice to realise sustainable growth. To these aims, the paper presents a model integrating production networks and sustainable freight transportation. The model includes Bill-of-Material constraints with alternative sources having different production and distribution costs. The objective function considers sourcing, production and transportation costs as well as environmental impacts of transport over a multimodal network. Due dates and time windows constraints for production and transportation management are included. Computational experiments are based on a real multimodal network. The optimisation model solved the case study instance. A sensitivity analysis proved the model robustness. The results demonstrate that the model can be effectively used in order to analyse costemissions trade-offs and the influence of links' capacity on emissions.
\end{abstract}

Keywords: Production Network, Transportation, Sustainability, Optimisation.

\section{Introduction and Background}

Competitiveness and sustainability are key drivers for designing and managing global production networks. Current challenges for networked production are then to stay competitive by optimising system-wide costs and service levels as well as to minimise negative impacts of freight transport on environment and society. These objectives are often separately pursued or only partially integrated in research approaches as well as in planning tools used by industry. It becomes imperative to completely include and widely spread in the industrial practice effective methods and tools dedicated to the simultaneous fulfilment of both business and sustainable growth targets. The 
aforementioned objectives should be pursued not only at strategic level (network design) but also in the management of day-to-day operations.

To these aims, the article presents a model integrating production networks and sustainable freight transportation. The optimisation model includes Bill-of-Material (BOM) constraints with alternative supply sources having different production and distribution costs. The objective function concurrently considers sourcing, production and transportation costs as well as the environmental impact of transport over a multimodal network. Multimodality is represented through a specific graph model. Due dates and time windows constraints for production and transportation management are considered. Computational experiments are based on a real multimodal network.

The integration of production and transportation networks has been tackled in several optimisation problems for the minimisation of supply, production, inventory, facility, and transportation costs by using formulations based on multi-commodity production-distribution [1]. Reviews and classifications cover the design process of production-distribution networks [2], as well as models and algorithms for production and outbound distribution scheduling in make-to-order production systems [3] and the integration of production and distribution problems in global supply chains [4]. Only a few research works include the freight flow constraints related to the BOM in the Mixed Integer Linear Programming (MILP) formulations that have been used [4], [5]. Only a few works attempted to simultaneously pursue routing and scheduling material flows while minimizing the environmental impacts [6], [7]. In these networked production scenarios little evidence has been identified about the sourcing options from alternative suppliers. Environmental sustainability has been generally addressed in terms of $\mathrm{CO}_{2}$ emissions as well as other impact parameters encompassed in the objective functions [6], [7], [8], [9].

MILP, multi-objective programming and other analytical models have been used for designing sustainable logistic networks considering, respectively, uncertainty factors [10], environmental performance and cost efficiency of the logistics networks [11] as well as carbon footprints in supply chains [12]. In these works, BOM constraints, sourcing options as well as transportation planning are not included.

In general, a multimodal transportation problem can be modelled through a multicommodity capacitated network design problem [13]. Nonetheless, another fundamental formulation is the multimodal, multiple capacity constraints, and multiple time windows transportation problem $(\mathrm{M}++\mathrm{P})$ [14]. This model constitutes the foundation of other research works that integrate short haul and long haul freight transportation problems [15].

On the basis of the literature review, it seems that research lines faced the management of freight flows across production and logistics networks with the prevalence of specific perspectives and focus, in production, transport or environmental impact performance, or partial combinations of these targets.

\section{Problem Definition and Methodology}

The problem is to determine the combination of production sites and multimodal routes over an integrated production and transportation network that concurrently 
minimises the total production and supply costs according to the BOM requirements, the transportation costs as well as the $\mathrm{CO}_{2}$ emissions of transportation means used to move materials, semi-finished and finished products. The time window constraints represent, at operational level, the output of a negotiated production scheduling process in a manufacturing network as performed in [16].

\subsection{Production Network Model}

The proposed approach considers a production network model in which components are produced and assembled in different plants in order to build the required final product. In the network depicted in Fig. 1a, coloured nodes represent production and assembly centres, while white nodes represent intermediate logistics nodes. When the customer in node 1 issues an order for a final product, a shipment for the final product is associated from the node 2 to the node 1 . Under the hypothesis of a "make-toorder" or "source-to-order" [17] production model, upon each request of final product $k$ from an origin $o_{k}$ (node 2 in the Fig. 1a) to a destination $d_{k}$, and under the hypothesis of zero inventories for intermediate and final products, correspondent orders for the components to be produced or assembled upstream in different locations are issued.

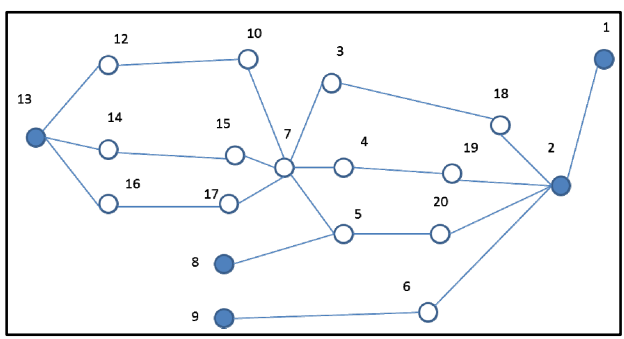

a. Production and logistics network

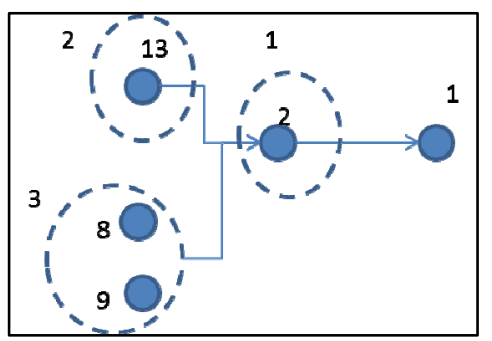

b. BOM with alternative sources

Fig. 1.

\subsection{Integration of BOM and Suppliers in the Production Network}

In order to consider the assembly relation among the different product flows in the network, a representation of the BOM must be associated to the production and logistics network. The production/assembly nodes in Fig. 1a are put in a "BOM relation" as in Fig. $1 \mathrm{~b}$ and must be considered in the mathematical model as flow and time constraints. The representation should consider also alternative sources for the same component in a specific position of the BOM.

\subsection{Integration of Transportation Impacts}

The approach considers multimodality in shipments. Transportation modes are drawn at graph level as done in [6], [14], [15]. Each physical arc is duplicated to represent a single mode of transport. Mode changes are modelled by duplicating nodes and drawing virtual arcs representing the modal change. This choice allows separating the costing and the sustainability considerations for each transportation mode. 
Different approaches are used for considering sustainability factors in logistics and manufacturing modelling. Sustainability factors are considered in our model at objective function level. The objective function must minimise both transport costs and emissions that are produced by vehicles. A sustainability factor comes from the $\mathrm{CO}_{2}$ emissions of each transportation vehicle. The emission factor $e_{i j}$ is associated to each $\operatorname{arc}(i, j) \in A$ of the network (i.e., to each transportation mode).

\subsection{Solution Approach}

As described in [13], a multimodal transportation problem can be modelled as a multicommodity capacitated network design problem defined on a directed graph $G=(N, A)$ where a set of commodities (shipments) $K$ have to be routed according to known demands and a set of facilities have to be installed on arcs (arc-based formulation). This model was used in different variants in order to solve transportation problems over integrated networks for short-haul and long-haul freight transport (see, for instance, [14], [15]). In the proposed approach, arcs and nodes are replicated in order to have a single arc for each multimodal transportation mean (e.g., a specific scheduled rail service). With respect to the mentioned formulations, $K$ commodities are originated from specific, different sources according to the hierarchical product decomposition included in the BOM. The synchronisation of inbound and outbound freight flows is imposed through appropriate constraints in order to make materials and components available in a production plant for the final assembly. Different transportation means are made accessible between each pair of nodes by replicating the arcs corresponding to each available transportation service. A transportation service is a capacitated, single modal transportation operation that can be carried out in a specific time window. The optimisation procedure simultaneously minimises both the sourcing costs and the transportation costs including the contribution of the environmental impact.

For each node $i \in N$ let $\Delta^{+}(i)$ the nodes $j \in N$ such that $(i, j) \in A$ and $\Delta^{-}(i)$, the nodes $j$ $\in N$ such that $(j, i) \in A . \bar{k} \in K$ be the shipment of the final product. Time windows are defined as $\left[a_{i}, b_{i}\right]$ for each $i \in N$ while $[E, L]$ is the earliness and lateness of the problem. Let moreover $s_{i}$ the service time at node $i$, for each $i \in N$; $t_{i j}$ the time needed to traverse an $\operatorname{arc}(i, j) \in A ; w_{i k}, i \in N, k \in K$ the arrival time at node $i$ for shipment $k ; x_{i j k},(i, j) \in A$, $k \in K$ equal to 1 if shipment $k$ uses arc $(i, j), 0$ otherwise. Other parameters of the model are: $M$, a large constants; $q_{k}$ the quantity of shipment $k \in K ; C_{i j}$ the capacity of $\operatorname{arcs}(i, j) \in$ $A ; c_{i j}$ the cost of traversing arc $(i, j) \in A ; f_{i}$ the activation cost of node $i \in N ; p_{i}$ the production cost of node $i \in N ; \alpha, \beta: \alpha+\beta=10$ the weights for cost and emission factors in objective function.

In the proposed model, the $k$ shipments are linked due to the BOM structural constraints. To this aim we define a set $B \subset K$ x $K$ containing the precedence constraints between shipments which define the BOM structure. A tuple $(h, k) \in B$ defines the shipment $h$ as a requirement (a material or a subassembly) for the shipment $k$ (a final product or a subassembly).

The model considers alternative sources (i.e., suppliers) for materials and assemblies. At each shipment $k \in \mathrm{K}$ is associated a subset of alternative origin nodes $O^{k} \subset N$ in which the shipment can be produced. $d^{k} k \in K$, is the destination node for 
shipment $k$ while $I^{k}$ are the subsets of intermediate nodes for shipment $k$ with $I=\cup I^{k}$. The following relation between origins and destinations can be stated:

if $(h, k) \in B=>\exists d \in O^{k}: d=d^{h}$.

Therefore, the origin of the shipment $k$ corresponds to the destination of the shipment $h$. At each node $i \in O=\cup_{k \in K} O^{k}$, a fixed activation cost $f_{i}$ and a variable production cost $p_{i}$ are defined, while a production cycle time for node $i$ is defined as $r_{i}$.

The integrated model can be formulated as follows:

$$
\begin{aligned}
& \min \alpha\left[\sum_{k \in K} \sum_{(i, j) \in A} c_{i j} x_{i j k}+\sum_{k \in K} \sum_{i \in O^{k}} \sum_{j \in \Delta^{+}(i)} q_{k} p_{i} x_{i j k}+\right. \\
& \left.\sum_{k \in K} \sum_{i \in O^{k}} \sum_{j \in \Delta^{+}(i)} f_{i} x_{i j k}\right]+\beta\left[\sum_{(i, j) \in A} \sum_{k \in K} e_{i j} x_{i j k}\right]
\end{aligned}
$$

subject to

$$
\begin{aligned}
& \sum_{j \in \Delta^{+}\left(o^{\bar{k}}\right)} x_{o^{\bar{k}} j \bar{k}}=1 \\
& \sum_{i \in \Delta^{-}\left(d^{\bar{k}}\right)} x_{i d^{\bar{k}} \bar{k}}=1 \\
& \sum_{i \in O^{k}} \sum_{j \in \Delta^{+}(i)} x_{i j k}-\sum_{i \in \Delta^{-}\left(d^{k}\right)} x_{i d^{k} k}=0, \quad \forall k \in K \backslash \bar{k} \\
& \sum_{j \in \Delta^{+}(i)} x_{i j k}-\sum_{j \in \Delta^{-}(i)} x_{j i k}=0, \quad \forall k \in K, \forall i \in I^{k} \\
& \sum_{i \in O^{h}} \sum_{j \in \Delta^{+}(i)} x_{i j h}-\sum_{j \in \Delta^{+}\left(d^{k}\right)} x_{d^{k} j k}=0, \quad \forall h \in K, k:(h, k) \in B \\
& w_{i k}+s_{i}+t_{i j k}-w_{j k} \leq\left(1-x_{i j k}\right) M \\
& \forall k \in K, \forall(i, j) \in A \\
& w_{i h}+s_{i}+r_{i}-w_{i k} \leq\left(1-\sum_{j \in \Delta^{-}(i)} x_{j i h}\right) M \\
& \forall h \in K, \forall i \in D^{h}, \forall h:(h, k) \in B \\
& a_{i}\left(\sum_{j \in \Delta^{+}(i)} x_{i j k}\right) \leq w_{i k} \leq b_{i}\left(\sum_{j \in \Delta^{+}(i)} x_{i j k}\right) \\
& \forall k \in K, \forall i \in N
\end{aligned}
$$




$$
\begin{aligned}
& E \leq w_{i k} \leq L, \quad \forall k \in K, \forall i \in\{O, D\} \\
& \sum_{k \in K} q_{k} \sum_{j \in \Delta^{+}(i)} x_{i j k} \leq C_{i j}, \quad \forall(i, j) \in A \\
& x_{i j k} \in\{0,1\}, \quad \forall k \in K, \forall(i, j) \in A
\end{aligned}
$$

The objective function (1) consists of four terms. The first term expresses the transportation cost. The second and third terms consider, respectively, production and activation cost, while the fourth term considers the emission factors (weighted with $\beta$, whereas the first three terms are weighted with $\alpha$ ). Constraints (2) - (5') characterize the flow to be followed by shipment $k$ without splits in the inner nodes. Constraints (5') states that a shipment $h$ is activated only if the related shipment $k$ is activated with $(h, k) \in B$. Constraints (6) - (8) guarantee schedule feasibility with respect to time considerations. The constraint (6') imposes time synchronization between shipments following the BOM definition. Constraint (9) guarantees feasibility with respect to capacity considerations while constraints (10) define the binary decision variables.

\section{Test Scenario and Experiment Design}

The network consists of facilities located in central and northern Italy. For the test purposes, a subset of facilities representing the major sources and destinations of freight flows has been selected. More specifically, they are the freight flows with the highest levels and importance travelling on the network from Lombardia and Piemonte regions (north-western Italy) to Lazio region (central Italy), through hubs located in Lombardia region (blue circle in Fig. 2) as well as Emilia Romagna region (green circle in Fig. 2).

According to the BOM representing, e.g., the assembly of kits for the telecom market, four facilities and nine modal transport connections are considered in the test instances. Transportation costs consist of a fixed and variable component dependent on the distance to be covered. The maximum distance from an origin to a destination is approximately $700 \mathrm{Km}$. Additional service times represent transhipment between different transport modes or vehicles. The transport modes considered are road and rail. The $\mathrm{CO}_{2}$ emissions are expressed in $\mathrm{Kg} \mathrm{CO}_{2} /$ Tonne- $\mathrm{Km}$.

The model has been also used to quantify the impact of links capacity on transportation costs and emissions. To this aim, restrictive link capacity constraints have been set for the connection of the shipments related to components and materials (not for the final product) and allowing flow splits ( $x_{i j k}$ not integer). Under these settings, the objective function can be used to evaluate investments in link capacity in order to reduce transportation costs and emissions. Regarding the solution approach to the presented optimisation problem, a solver has been used. 


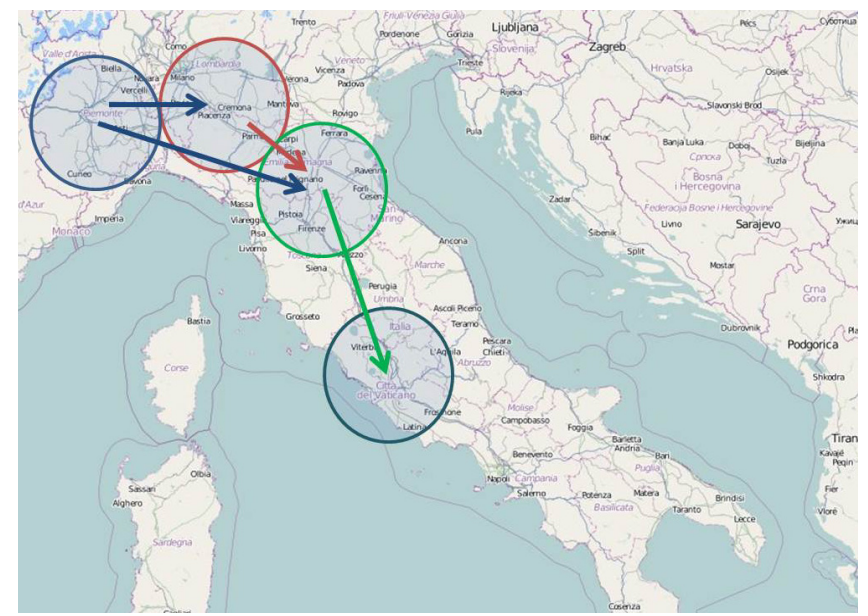

Fig. 2. Test scenario

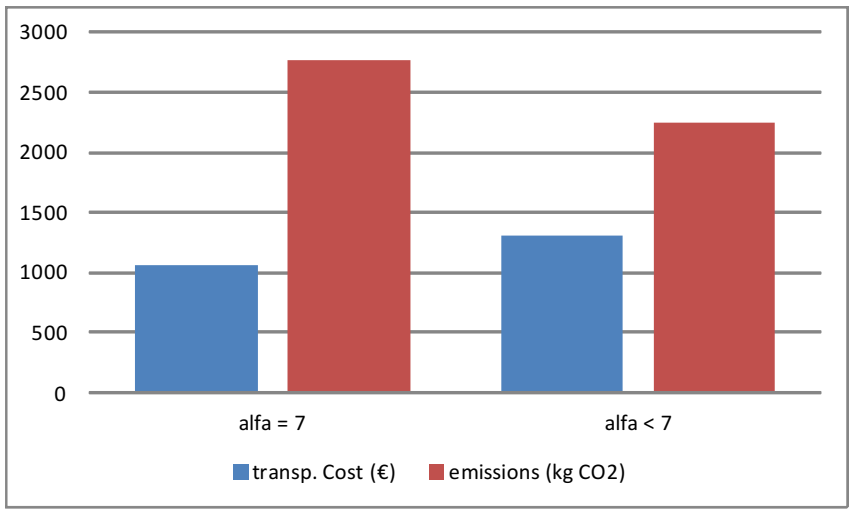

Fig. 3. Transportation cost and emissions

\section{Computational Results and Discussion}

The model has been implemented on IBM ILOG CPLEX Optimization Studio ${ }^{\circledR}$. The case study presented in this paper consists of a production network of 19 nodes, 22 arcs, 3 shipments, with 1 shipment having 2 alternative sources. This case study was solved in 0.37 seconds on a windows PC with Intel ${ }^{\circledR}$ Core $^{\mathrm{TM}} 2$ Duo T5750 $2.00 \mathrm{GHz}$ processor with 4 GB RAM. In the case study, the incidence of transportation costs on the production costs is lower than $5 \%$. Moreover, since the alternative sources have similar production costs, the solver selected the node 9 (see Fig. 1b) as the best source alternative. Different sensitivity analyses have been conducted. The most important is related to the tuning of the weights for transportation costs and emission factors ( $\alpha$ and $\beta$ ). The results (see exhibit in Fig. 3) demonstrate that it is possible to obtain a maximum reduction in $\mathrm{CO}_{2}$ emissions of $19 \%$ if compared to the case where only 
transportation and production costs are considered. On the other hand, this emission reduction corresponds to an increase in transportation costs approximately equal to $23 \%$. The model can be effectively used in order analyse the desiderated trade-off between cost and $\mathrm{CO}_{2}$ emission.

Concerning the experiments related to the modification of capacity of links and implications in terms of environmental impacts, Fig. 4 shows a simulation which demonstrates the tight connection between link capacity and $\mathrm{CO}_{2}$ emissions. More specifically, this experiment suggests that the introduction of more stringent constraints on the capacity of the links leads to the selection of alternatives paths over the network in order to move the requested quantities. In fact, the increased material flows entail an increase in the emissions due to solutions that tend to allocate the shipments to modes with higher environmental impacts (e.g., road mode) or to paths with longer distances. Therefore, this type of experiment can be used for evaluating potential adjustments of link capacity on the most critical arcs. This could be carried out both in terms of (i) further development of logistics and transportation infrastructures as well as (ii) increase in the number of transportation services with lower environmental impacts on specific paths.

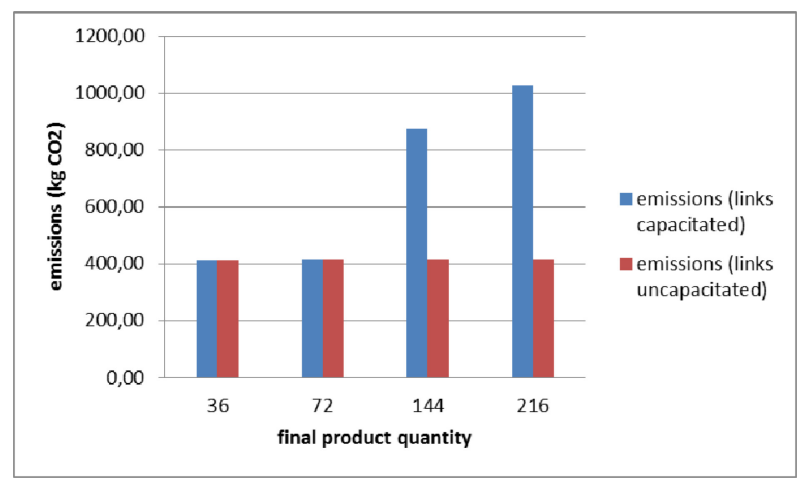

Fig. 4. Emissions and link capacity constraints

\section{$5 \quad$ Conclusions and Future Research}

This work presented a methodology integrating production and sustainable freight transportation in a production network including BOM constraints and multimodal connections. A MILP model effectively solved the presented case study and the sensitivity analysis demonstrated the model robustness. Moreover, the model can support the evaluation of the capacity of the network or sub-networks that may lead to lower environmental impacts of freight transport operations.

The main limitations of this work concern the solution of large instances and the inclusion of capacity constraints at sourcing level together with issues related to make-to-order systems. Regarding the former aspect, the model can encounter difficulties in solving large instances over highly detailed networks due to the complex graph representation. This entails the need to elaborate in future research 
tasks a smarter solution approach able to reduce the complexity of the problem or by decomposing the model, as performed in [18]. Concerning the latter limitation aspect of the work, the study has been conducted by focusing on a robust, integrated model with a degree of complexity compliant with the purpose of the development step of this research. The choice of the production model setting has been made in order to limit, at the current stage of the research, the complexity of the inclusion in the model of inventory level issues. The current research stage considers the hypotheses that the required materials or components are available at the suppliers' sites and no inventories are available at the production and assembly sites of the final products. Therefore, future developments of the research may consider the increase of the model complexity degree by including further elements (e.g., in terms of supply capacity, production lead-times, depth of penetration of customer orders) useful for improving the decision-making in make-to-order production models (see, for instance, [19], [20], [21]).

Future works may also consider the use of cross docks, inventory as well as reverse flows of materials in closed loop supply chains. From the solution approach viewpoint, the implementation of metaheuristics or alternative methodological approaches will be possibly considered.

\section{References}

1. Geoffrion, A.M., Graves, G.W.: Multicommodity Distribution System Design by Benders Decomposition. Manage. Sci. 20, 822-844 (1974)

2. Martel, A.: The Design of Production-Distribution Networks: A Mathematical Programming Approach. In: Geunes, J., Pardalos, P.M. (eds.) Supply Chain Optimization, Applied Optimization, vol. 98, pp. 265-305. Springer, New York (2005)

3. Chen, Z.L.: Integrated Production and Outbound Distribution Scheduling: Review and Extensions. Oper. Res. 58(1), 130-148 (2010)

4. Bilgen, B., Ozkarahan, I.: Strategic, Tactical and Operational Production-Distribution Models: A Review. Int. J. Technol. Manage. 28(2), 151-171 (2004)

5. Yan, H., Yu, Z., Cheng, T.C.E.: A Strategic Model for Supply Chain Design with Logical Constraints: Formulation and Solution. Comput. Oper. Res. 30, 2135-2155 (2003)

6. Confessore, G., Galiano, G., Liotta, G., Stecca, G.: A Production and Logistics Network Model with Multimodal and Sustainability Considerations. In: Proceedings of the 8th CIRP Conference on Intelligent Computation in Manufacturing Engineering, Ischia, Italy (2012)

7. Le, T.P.N., Lee, T.R.: Model Selection with Considering the $\mathrm{CO} 2$ Emission along the Global Supply Chain. J. Intell. Manuf. 24(4), 653-672 (2013)

8. Dotoli, M., Fanti, M.P., Meloni, C., Zhou, M.C.: Design and Optimization of Integrated ESupply Chain for Agile and Environmentally Conscious Manufacturing. IEEE T. Syst. Man. Cy. A. 36(1), 62-75 (2006)

9. Wang, F., Lai, X., Shi, N.: A Multi-Objective Optimization for Green Supply Chain Network Design. Decis. Support Syst. 51, 262-269 (2011)

10. Lee, D.H., Dong, M., Bian, W.: The Design of Sustainable Logistics Network under Uncertainty. Int. J. Prod. Econ. 128, 159-166 (2010) 
11. Quariguasi Frota Neto, J.Q., Bloemhof-Ruwaard, J.M., van Nunen, J.A.E.E., van Heck, E.: Designing and Evaluating Sustainable Logistics Networks. Int. J. Prod. Econ. 111, 195-208 (2008)

12. Sundarakani, B., de Souza, R., Goh, M., Wagner, S.M., Manikandan, S.: Modeling Carbon Footprints across the Supply Chain. Int. J. Prod. Econ. 128, 43-50 (2010)

13. Gendron, B., Crainic, T., Frangioni, A.: Multicommodity Capacitated Network Design. In: Sansò, B., Soriano, P. (eds.) Telecommunications Network Planning, pp. 1-19. Kluwer, Boston (1998)

14. Moccia, L., Cordeau, J.F., Laporte, G., Ropke, S., Valentini, M.P.: Modeling and Solving a Multimodal Transportation Problem with Flexible-Time and Scheduled Services. Networks 57(1), 53-68 (2011)

15. Galiano, G., Kaihara, T., Liotta, G., Stecca, G.: A Comprehensive Model for Short-Haul and Long-Haul Transportation. In: Proceedings of the 8th IEEE International Conference on Industrial Informatics, Osaka, Japan, pp. 940-944. IEEE, Piscataway (2010)

16. Kaihara, T., Yao, Y., Fujii, N.: Interdivisional Production Scheduling with Social Negotiation Mechanism. CIRP Ann.-Manuf. Techn. 60, 461-464 (2011)

17. Arlbjørn, S.J., Haas, H., Mikkelsen, S.O., Zachariassen, F.: Supply Chain Management: Sources for Competitive Advantages. Academica, Aarhus (2010)

18. Kaihara, T., Kurose, S., Fujii, N.: A Proposal on Optimized Scheduling Methodology and its Application to an Actual-Scale Semiconductor Manufacturing Problem. CIRP Ann.Manuf. Techn. 61, 467-470 (2012)

19. Dekkers, R.: Engineering Management and the Order Entry Point. Int. J. Prod. Res. 44, 4011-4025 (2006)

20. Rudberg, M., Wikner, J.: Mass Customization in Terms of the Customer Order Decoupling Point. Prod. Plan. Control 15, 445-458 (2004)

21. Olhager, J.: Strategic Positioning of the Order Penetration Point. Int. J. Prod. Econ. 85, 319-329 (2003) 